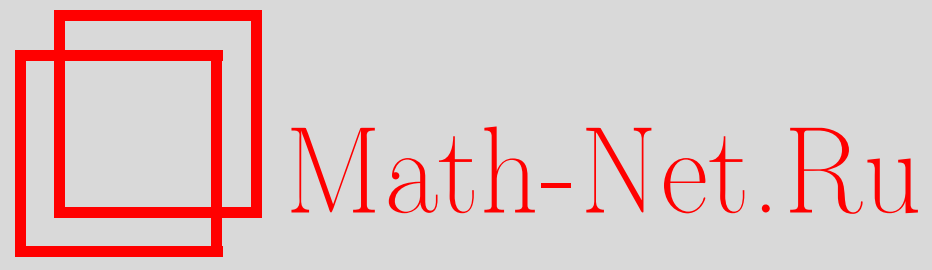

С. А. Смолянский, А. В. Прозоркевич, М. Бониц, Некоторые проблемы кинетического описания вакуумного рождения частиц в сильных полях, Вестн. Сам. гос. техн. ун-та. Сер. Физ.-мат. науки, 2013, выпуск 2(), 206-214

DOI: https://doi.org/10.14498/vsgtu1187

Использование Общероссийского математического портала Math-Net.Ru подразумевает, что вы прочитали и согласны с пользовательским соглашением

http://www.mathnet.ru/rus/agreement

Параметры загрузки:

IP: 3.80 .253 .173

26 апреля 2023 г., $10: 21: 44$

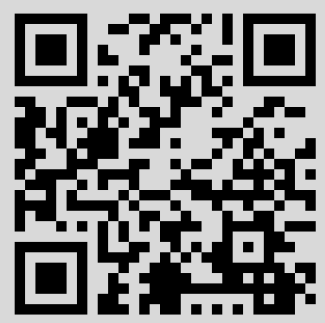




\title{
НЕКОТОРЫЕ ПРОБЛЕМЫ КИНЕТИЧЕСКОГО ОПИСАНИЯ ВАКУУМНОГО РОЖДЕНИЯ ЧАСТИЦ В СИЛЬНЫХ ПОЛЯХ
}

\author{
С. А. Смолянский ${ }^{1}$, А.В. Прозоркевич ${ }^{1}$, М. Боний \\ 1 Саратовский государственный университет им. Н. Г. Чернышевского \\ (национальный исследовательский университет), механико-математический факультет, \\ Россия, 410012, Саратов, ул. Астраханская, 83. \\ 2 Институт теоретической физики и астрофизики, университет Кристиана-Альбрехта, \\ Германия, 24098, Киль, Лейбниц штр. 15. \\ E-mails: smol@sgu.ru, avproz@yandex.ru, bonitz@theo-physik.uni-kiel.de
} Обсуждаются некоторые актуальные проблемы в теории вакуумного рождения
частич в сильном внешнем поле.

Ключевые слова: вакуумное рождение пар, кинетическое уравнение, теория возмущений.

Введение. Исследование квантовых эффектов в сильных внешних полях имеет долгую историю. Практически сразу после появления уравнения Дирака был обнаружен парадокс Клейна [1], который получил впоследствии адекватное истолкование как процесс вакуумного рождения пар [2]. В 1951 г. Ю. Швингер [3] получил точный результат для вероятности рождения электрон-позитронных пар в постоянном электрическом поле, который позднее стали называть формулой Швингера, а само явление вакуумного рождения в поле - механизмом Швингера. Характерный масштаб напряженности электрического поля, определяемый формулой Швингера (критическое поле), оказался настолько велик, $E_{c r} \sim 10^{16} \mathrm{~B} / \mathrm{cm}$, что экспериментальная проверка этого фундаментального эффекта остается недоступной до сих пор.

Ситуация стала заметно меняться только в последние годы в связи со стремительным развитием лазерных технологий в такой области интенсивности поля $\left(\sim 10^{20} \mathrm{~B} \mathrm{~T} / \mathrm{cm}^{2}\right)$, что можно говорить о возникновении нового направления на стыке лазерной физики и физики высоких энергий - релятивистской лазерной оптики $[4,5]$. Настоящий технологический прорыв произошел в результате реализации метода «chirped pulse amplification» [6], после чего лазеры быстро достигли уровня интенсивности $\sim 10^{18} \mathrm{~B}$ т $/ \mathrm{cm}^{2}$. В полях таких лазеров электроны достигают релятивистских энергий и могут инициировать неупругие элементарные реакции. Тем временем предлагаются новые технологии, например «coherent harmonic focusing» [7], с помощью которых становится принципиально достижимым уровень интенсивности лазерного поля $\sim 10^{29} \mathrm{~B}$ т см $^{2}$ (швингеровский предел). В этом случае процесс рождения пар идет настолько интенсивно, что такое поле не может существовать на макроскопических временах. Но даже на микроскопических временах ток рождающихся пар генерирует собственное поле, сравнимое по величине с критическим («back-reaction»-эффект), поэтому внешнее поле не может поддерживаться по энергетическим соображениям. Поэтому критическое поле

Станислав Александрович Смолянский (д.ф.-м.н., проф.), профессор, каф. теоретической физики. Александр Васильевич Прозоркевич (д.ф.-м.н., доц.), профессор, каф. общей физики. Михаэль Бонии, профессор, каф. статистической физики. 
определяет естественный предел применимости КЭД по величине поля [8].

C точки зрения эксперимента, в недалеком будущем станет возможным изучение целого ряда нелинейных вакуумных эффектов, таких как рождение пар, двойное лучепреломление, рассеяние света на свете, расщепление фотона, генерация высших гармоник и т.п. В связи с этим в последние годы наблюдается новая волна интереса к изучению нелинейных вакуумных явлений в сильных полях.

Первые теоретические оценки вероятности рождения пар в лазерном поле были сделаны уже в 1969 г. [9], позднее они неоднократно уточнялись различными методами и в различных приближениях [10-12]. Основное продвижение в теории было связано с переходом от случая постоянного поля к электромагнитным полям с произвольной зависимостью от времени. Были предложены различные методы, среди которых можно выделить S-матричный метод (Н. Б. Нарожный, А. И. Никишов, В. И. Ритус; В. Г. Багров, Д. М. Гитман, Е. С. Фрадкин, Ш. М. Шварцман), метод мнимого времени (квазиклассический подход, В. С. Попов), метод диагонализации гамильтониана (А. А. Гриб, С. Г. Мамаев, В. М. Мостепаненко, В. М. Фролов), осцилляторное представление (В. Н. Первушин). Наиболее общим на сегодняшний день является метод функций Вигнера [13], пригодный для полей с произвольной пространственно-временной зависимостью.

С помощью этих методов удалось рассмотреть достаточно широкий класс электромагнитных полей: плоские волны различной поляризации, комбинации плоских волн и постоянных полей, однородные поля с произвольной зависимостью от времени. Наиболее полное описание удается получить при наличии точных решений уравнения Дирака, такие решения известны для случая плоской волны [14] и для импульсного однородного поля [15]. Для произвольного однородного переменного поля задача сводится к решению одномерного осцилляторного уравнения с комплексной частотой. Тем не менее доступный на сегодня спектр точных решений не позволяет учесть одновременно два ключевых условия для лазерного поля: ограниченность поля как во времени, так и в пространстве. Поэтому широко используются численные методы.

Основные усилия теоретиков были направлены на поиск асимптотических решений при $t \rightarrow+\infty$, когда внешнее поле выключается. В такой постановке вероятность выживания пары в подкритических полях $E \ll E_{c r}$ исчезающе мала. Для полей, действующих в течение ограниченного интервала времени $\tau$, важным управляющим параметром становится отношение комптоновского времени $\tau_{c} \mathrm{~K} \tau$ : если $\tau_{c} / \tau \ll 1$ и $E \ll E_{c r}$, то во время действия поля может рождаться заметное количество пар квазичастиц, однако они успевают полностью аннигилировать за время выключения поля. Это подсказывает другую возможную тактику эксперимента: регистрация вторичных продуктов элементарных реакций квазичастичных пар, например, аннигиляционных фотонов. Это направление интенсивно развивается в последние годы [16]. Некоторые предварительные оценки двухфотонной аннигиляции [17] планируется проверить в эксперименте на одном из самых мощных действующих оптических лазеров Astra Gemini, Rutherford Appleton Laboratory, UK.

Наиболее интересным теоретическим результатом последних лет является предсказание недостижимости швингеровского предела из-за развития каскадных реакций, разрушающих лазерный импульс уже при $E \sim 0,01 E_{c r}$ 
[18-20]. Точные количественные оценки такого рода для реальных полей вряд ли возможны пока даже на суперкомпьютерах, поэтому интрига, скорее, всего сохранится до реализации проектов «The Extreme Light Infrastructure»[22].

По мере развития теории вакуумного рождения оказалось, что ее применимость не ограничивается квантовой электродинамикой. После появления ускорителей тяжелых ионов эксперименты по столкновениям релятивистских ядер стали одним из основных источников информации для фундаментальной физики. Такие столкновения порождают многочастичную сверхплотную неравновесную систему, которая быстро $\left(\tau \approx 10^{-23} \mathrm{c}\right)$ эволюционирует, испытывая фазовые переходы и проходя стадию образования кварк-глюонной плазмы. Все это заканчивается стадией адронизации, множественного рождения и вылета различных типов частиц. Швингеровский механизм оказался полезным инструментом для описания ранней стадии этого процесса.

Еще одно применение теории вакуумного рождения обнаружилось в моделях со спонтанным нарушением симметрии, например, калибровочной или киральной, где фазовые переходы сопровождаются изменением эффективной массы частиц. Это изменение запускает новый механизм генерации пар, отличающийся от полевого. Кроме того, учет конечных продольных размеров цветовой трубки, границы которой быстро расширяются, приводит к вакуумному рождению пар, которое можно рассматривать как динамический аналог классического эффекта Казимира, впервые рассмотренного Ю. Швингером [24]. Таким образом, модель цветовых трубок может служить основой для построения комбинированного описания вакуумного рождения под действием трех различных конкурирующих механизмов.

1. Приближение однородного поля. Практически все результаты теории вакуумного рождения получены для пространственно-однородных полей. В этом случае можно разделить переменные в уравнении Дирака и свести задачу о рождении пар к задаче о параметрическом возбуждении квантового осциллятора с переменной (комплексной) частотой [25]. Характерное расстояние, на котором рождается пара, определяется выражением

$$
l=\frac{\lambda}{\pi} \operatorname{arctg}\left(\frac{\nu}{m} \frac{E_{c}}{E}\right),
$$

где $\lambda$ - длина волны, $\nu$ - частота поля. Для оптических лазеров $\nu / m \sim 10^{-6}$ условие применимости однородного приближения $l \ll \lambda$ будет выполняться при $E>10^{-5} E_{c}$, что как раз соответствует области релятивистской лазерной оптики [5]. Более уязвимым является пренебрежение переменным магнитным полем, которое основано на предположении о том, что частицы рождаются в состояния с малыми импульсами.

Обычно используется однородное поле электрического типа

$$
\mathbf{E}=\mathbf{E}(t), \quad \mathbf{B}=0,
$$

которое получило условное название «лазерный свет» [26]. Известно [3], что в электромагнитном поле, у которого оба инварианта равны нулю $\left(\mathbf{E}^{2}-\mathbf{B}^{2}=0\right.$, $\mathbf{E} \cdot \mathbf{B}=0$ ), вакуумное рождение пар невозможно (например, плоская волна). Поле, образующееся в лазерном фокусе, близко по структуре к такой конфигурации [27], поэтому в обычном лазерном луче рождение пар подавлено при любой интенсивности. Это ограничение можно обойти в конфигурации двух 
встречных когерентных пучков. Предполагается, что поле, образующееся в пучности стоячей волны, будет однородным полем электрического типа, по крайней мере в области с линейным размером, много меньшим длины волны. Однако такое поле не является решением уравнений Максвелла в пустом пространстве и не отражает структуру реальных гауссовых пучков.

Условием квазиклассичности электрического поля является неравенство $E \gg 1 / \Delta t^{2}[28]$, где $\Delta t-$ характерный масштаб усреднения. Для периодического поля $\Delta t \sim 1 / \nu$, поэтому условие квазиклассичности можно представить в виде

$$
\frac{E}{E_{c}} \gg e\left(\frac{\nu}{m}\right)^{2}
$$

Это условие хорошо выполняется для действующих тераваттных оптических лазеров и строящихся рентгеновских лазеров на свободных электронах [29].

Нахождение функции распределения квазичастиц $f(\mathbf{p}, t)$ в поле $(1)$ в любой момент времени сводится к решению системы трех обыкновенных линейных дифференциальных уравнений [30,31], в которые импульс р (канонический) входит как параметр:

$$
\dot{f}=\frac{1}{2} \Delta u, \quad \dot{u}=\Delta(1-2 f)-2 \varepsilon v, \quad \dot{v}=2 \varepsilon u,
$$

где $u(\mathbf{p}, t), v(\mathbf{p}, t)$ - вспомогательные функции, $\Delta=e E \varepsilon_{\perp} / \varepsilon^{2}, \varepsilon_{\perp}=\sqrt{m^{2}+p_{\perp}^{2}}$, $\varepsilon(\mathbf{p}, t)=\sqrt{\varepsilon_{\perp}^{2}+\left[p_{\|}-e A(t)\right]^{2}}, E(t)=-\dot{A}(t)$. Исключение вспомогательных функций $u, v$ приводит к замкнутому уравнению для функции распределения:

$$
\dot{f}(\mathbf{p}, t)=\frac{1}{2} \Delta(\mathbf{p}, t) \int_{0}^{t} d t^{\prime} \Delta\left(\mathbf{p}, t^{\prime}\right)\left[1-2 f\left(\mathbf{p}, t^{\prime}\right)\right] \cos 2\left\{\int_{t^{\prime}}^{t} d \tau \varepsilon(\mathbf{p}, \tau)\right\} .
$$

Это уравнение немарковского типа с быстро осциллирущим аргументом косинуса, препятствующим выделению медленно меняющейся части функции распределения по методу Боголюбова-Митропольского.

В наиболее интересной области параметров (оптические лазеры) вычислительная задача (2) оказывается экстремально жесткой из-за наличия двух временных масштабов, различающихся на много порядков: комптоновского времени $\tau_{c} \sim 1 / m$, определяющего время рождения (аннигиляции) пары, и времени действия лазерного поля $\tau \sim 100 / \nu$.

Решения системы (2) демонстрируют очень сложную зависимость от импульса. В отличие от большинства феноменологических подходов, где предполагается, что частицы рождаются в покое, система (2) генерирует частицы с любыми импульсами, спектр которых затухает степенным образом. В зависимости от формы внешнего поля в процессе возбуждения вакуума могут формироваться очень сложные и разнообразные, сильно неравновесные спектры. Сложная форма импульсного спектра частиц может существенно влиять на сечения элементарных реакций и на развитие каскадов, а также иметь определенные наблюдаемые проявления.

Наиболее ярким отличием спектра рожденных полем частиц от равновесного является его существенная немонотонность. Эта особенность была 
отмечена еще в работах $[32,33]$ для случая периодического поля, а позднее изучена подробнее для гармонического поля с гауссовой огибающей в работе [34]. Аналитически связь этого явления с линиями Стокса системы (2) была установлена в [35].

Следует отметить, что этот эффект не описывается полуклассическими подходами $[10,12]$. Единственное известное точное решение для поля Заутера также порождает гладкий импульсный спектр [15]. Для возникновения немонотонной деформации $f(\mathbf{p}, t)$ необходимо наличие в спектре внешнего поля изолированных зон разного масштаба, как, например, для радиоимпульса (целое число периодов гармонического поля). Реальное лазерное поле также имеет в спектре два различных временных масштаба, поэтому вопрос о форме $f(\mathbf{p}, t)$ имеет важное практическое значение.

Численное решение (2) показывает, что деформация $f(\mathbf{p}, t)$ гораздо более выражена в промежуточных состояниях, когда превалируют эффекты поляризации вакуума. Это должно иметь существенное значение для скоростей элементарных реакций квазичастиц и развития каскадов [20]. Наиболее выражена деформация $f(\mathbf{p}, t)$ в случае радиоимпульса, где формируется сложная структура кольцевых ячеек различной плотности. При этом в начале процесса рождения форма $f(\mathbf{p}, t)$ всегда гладкая с одним максимумом и степенными «хвостами». Такое поле относится к классу неадиабатических. Именно оно используется в полуклассическом подходе [10,12] в сочетании в предельным переходом $N \rightarrow \infty$, после чего получается гладкая функция распределения. Очевидно, что при такой процедуре теряется некоторая существенная информация о спектре частиц после выключения поля.

Усложнение структуры функции распределения приводит к росту энтропии [21], который, однако, также имеет немонотонный характер. Тем не менее, если рассматривать действие импульса внешнего поля, то можно констатировать увеличение энтропии в конечном состоянии, где остаются реальные частицы, по сравнению с начальным состоянием до включения поля.

2. Неоднородное поле. Учет пространственной неоднородности поля является одной из актуальных задач теории вакуумного рождения. Соответствующие уравнения в формализме одновременных функций Вигнера были получены ещё в 1991 г. [13], однако первые попытки их решения были предприняты только сейчас [36].

В этом формализме используются бинарные комбинации полных решений уравнения Дирака без их вторичного квантования, что позволяет обойти проблему диагонализации гамильтониана во внешнем поле. Согласно [13], функция Дирака-Гейзенберга-Вигнера $W(\mathbf{r}, \mathbf{q}, t)$ определяется выражениeM

$$
\begin{aligned}
W_{\alpha \beta}(\mathbf{r}, \mathbf{q}, t)=-\frac{1}{2} \int d^{3} s \exp ( & -i \mathbf{q} \cdot \mathbf{s}-i \varphi) \times \\
& \times\left\langle\Phi\left|\left[\hat{\Psi}_{\alpha}(\mathbf{r}+\mathbf{s} / 2, t), \hat{\Psi}_{\beta}^{\dagger}(\mathbf{r}-\mathbf{s} / 2, t)\right]\right| \Phi\right\rangle,
\end{aligned}
$$

где $\hat{\boldsymbol{\Psi}}$ - квантованные дираковские операторы, $\Phi$ - произвольное чистое состояние, а $\varphi$ - фаза, обеспечивающая калибровочную инвариантность, кото- 
рая определяется линейным интегралом от векторного потенциала $\mathbf{A}$ :

$$
\varphi=e \int_{-1 / 2}^{1 / 2} d \lambda \mathbf{s} \cdot \mathbf{A}(\mathbf{r}+\lambda \mathbf{s}, t) .
$$

В отличие от (2) здесь используется кинематический импульс $\mathbf{q}=\mathbf{p}-e \mathbf{A}$.

В общем случае уравнение движения для $W(\mathbf{r}, \mathbf{q}, t)$ представляет собой матричное интегро-дифференциальное уравнение, которое можно свести к локальной (дифференциальной) форме в случае медленно меняющихся (на комптоновских масштабах) электромагнитных полей. После разложения (3) по базису алгебры матриц Дирака получается 16 уравнений в частных производных для компонент, 4-х скаляров $f_{0}, f_{1}, f_{2}, f_{3}$ и 4-х векторов $\mathbf{g}_{0}, \mathbf{g}_{1}, \mathbf{g}_{2}, \mathbf{g}_{3}$ :

$$
\begin{array}{rlrl}
D_{t} f_{0}+\mathbf{D} \cdot \mathbf{g}_{1} & =0, & & D_{t} \mathbf{g}_{0}+\mathbf{D} f_{1}-2 \mathbf{p} \times \mathbf{g}_{1}=0, \\
D_{t} f_{1}+\mathbf{D} \cdot \mathbf{g}_{0} & =-2 m f_{2}, & & D_{t} \mathbf{g}_{1}+\mathbf{D} f_{0}-2 \mathbf{p} \times \mathbf{g}_{0}=-2 m \mathbf{g}_{2}, \\
D_{t} f_{2}+2 \mathbf{p} \cdot \mathbf{g}_{3} & =2 m f_{1}, & & D_{t} \mathbf{g}_{2}+\mathbf{D} \times \mathbf{g}_{3}+2 \mathbf{p} f_{3}=2 m \mathbf{g}_{1}, \\
D_{t} f_{3}-2 \mathbf{p} \cdot \mathbf{g}_{2}=0, & D_{t} \mathbf{g}_{3}-\mathbf{D} \times \mathbf{g}_{2}-2 \mathbf{p} f_{2}=0,
\end{array}
$$

где $D_{t}=\partial_{t}+e \mathbf{E}(\mathbf{r}, t) \cdot \partial_{p}, \mathbf{D}=\nabla+e \mathbf{B}(\mathbf{r}, t) \times \partial_{p}$. В [13] было показано, что система (4) переходит в (2) в случае пространственно однородного электрического поля (1).

Численное решение системы (4) для реальных полей, по-видимому, недоступно даже для современных суперкомпьютеров. Так, по оценкам [37], для реализации минимальной сеточной схемы потребуется не менее 10 ТБ памяти. Поэтому в качестве первого шага авторы [36] редуцировали систему (4) на случай одномерной электродинамики. Было показано, что локализация поля в пространстве

$$
E(x, t)=E_{0} \exp \left(-\frac{x^{2}}{2 \lambda^{2}}\right) \operatorname{sech}^{2}\left(\frac{t}{\tau}\right)
$$

приводит к новым эффектам по сравнению с однородным полем, например, группировке пар в импульсном пространстве при уменьшении $\lambda$ и подавлению рождения при $\lambda \rightarrow 1 / m$.

Заключение. Учет пространственной неоднородности и вторичных реакций квазичастиц представляет собой наиболее актуальную задачу теории вакуумного рождения на ближайшие годы. Это означает переход от тривиальной одночастичной кинетики к квантовой теории релятивистской плазмы в сильном внешнем поле. Подход, основанный на анализе цепочки уравнений Боголюбова, обеспечивает достаточно строгое описание таких систем. Первый шаг в этом направлении был сделан в работе [38], где была изучена однофотонная аннигиляция в квазичастичной плазме.

\section{БИБЛИОГРАФИЧЕСКИЙ СПИСОК}

1. O. Klein, "Die Reflexion von Elektronen an einem Potentialsprung nach der relativistischen Dynamik von Dirac" // Z. Phys., 1929. Vol. 53. Pp. 157-165.

2. А. И. Никишов, "Образование пар постоянным внешним полем" // ЖЭТФ, 1969. Т. 57, № 4. C. 1210-1216; англ. пер.: A. I. Nikishov, "Pair Production by a Constant External Field" // Sov. J. Exp. Theor. Phys., 1970. Vol.30, no. 4. Pp. 660-662.

3. J. Schwinger, "On Gauge Invariance and Vacuum Polarization" // Phys. Rev., II. Ser., 1951. Vol. 82. Pp. 664-679. 
4. A. V. Borovski, A. L. Galkin, O. B. Shiryaev, T. Auguste, Laser physics at relativistic intensities. Berlin: Springer, 2003. 218 pp.

5. G. Mourou, T. Tajama, S. V. Bulanov, "Optics in the relativistic regime"// Rev. Mod. Phys., 2006. Vol. 78, no. 2. Pp. 309-371.

6. D. Strickland, G. Mourou, "Compression of amplified chirped optical pulses" // Opt. Commun., 1985. Vol. 56, no. 3. Pp. 219-221.

7. S. Gordienko, A. Pukhov, O. Shorokhov, T. Baeva, "Coherent Focusing of High Harmonics: A New Way Towards the Extreme Intensities"// Phys. Rev. Lett., 2005. Vol.94, no. 10, 103903. 4 pp.

8. N. Bohr, "Faraday lecture. Chemistry and the quantum theory of atomic constitution" // J. Chem. Soc., 1932. Pp. 349-384.

9. Ф. В. Бункин, И. И. Тугов, "О возможности рождения электронно-позитронных пар в вакууме при фокусировке лазерного излучения" // Докл. АН СССР, 1969. Т. 187, № 3. C. 541-544. [F. V. Bunkin, I. I. Tugov, "The possibility of electron-positron pair production in vacuum when laser radiation is focussed" // Dokl. AN SSSR, 1969. Vol. 187, no. 3. Pp. 541544].

10. E. Brezin, C. Itzykson, "Pair production in vacuum by an alternating field" // Phys. Rev. D, 1970. Vol. 2, no. 7. Pp. 1191-1199.

11. M. S. Marinov, V. S. Popov, "Electron-Positron Pair Creation from Vacuum Induced by Variable Electric Field" // Fortsch. Phys., 1977. Vol. 25. Pp. 373-400.

12. В. С. Попов, "О швингеровском механизме рождения электрон-позитронных пар из вакуума полем оптических и рентгеновских лазеров" // Писъма в ЖЭТФ, 2001. Т. 74, №3. C. 151-156; англ. пер.: V. S. Popov, "Schwinger mechanism of electron-positron pair production by the field of optical and X-ray lasers in vacuum" // J. Exp. Theor. Phys., 2001. Vol. 74, no. 3. Pp. 133-138.

13. I. Bialynicki-Birula, P. Górnicki, J. Rafelski, "Phase-space structure of the Dirac vacuum" // Phys. Rev. D, 1991. Vol.44, no.6. Pp. 1825-1835.

14. D. $M$ Wolkow, "Über eine Klasse von Lösungen der Diracschen Gleichung" // Z. f. Physik, 1935. Vol. 94, no. 3-4. Pp. 250-260.

15. Н. Б. Нарожный, А. И. Никишов, "Простейшие процессы в электрическом поле, порождающем пары” // Яд. физ., 1970. Т. 11, №5. С. 1072-1077; англ. пер.: N. B. Narozhnyi, A. I. Nikishov, "The Simplist processes in the pair creating electric field" // Sov. J. Nucl. Phys.. Vol. 11, no. 5. Pp. 596.

16. A. Di Piazza, C. Müller, K. Z. Hatsagortsyan, C. H. Keitel, "Extremely high-intensity laser interactions with fundamental quantum systems" // Rev. Mod. Phys., 2012. Vol. 84, no. 3. Pp. 1177-1228, arXiv: 1111.3886 [hep-ph].

17. G. Gregori, D. B. Blaschke, P. P. Rajeev, H. Chen, R. J. Clarke, T. Huffman, C. D. Murphy, A. V. Prozorkevich, C. D. Roberts, G. Röpke, S. M. Schmidt, S. A. Smolyansky, S. Wilks, R. Bingham, "A proposal for testing subcritical vacuum pair production with high power lasers" // High Energy Density Phys., 2010. Vol.6, no. 2. Pp. 166-170, arXiv: 1005.3280 [hep-ph].

18. A. R. Bell, J. G. Kirk, "Possibility of Prolific Pair Production with High-Power Lasers" // Phys. Rev. Lett., 2008. Vol. 101, no. 20, 200403. 4 pp., arXiv: 0808.2107 [hep-ph].

19. J. G. Kirk, A. R. Bell, I. Arka, "Pair production in counter-propagating laser beams" // Plasma Phys. Control. Fusion, 2009. Vol. 51, no. 8, 085008, arXiv: 0905.0987 [hep-ph].

20. A. M. Fedotov, N. B. Narozhny, G. Mourou, G. Korn, "Limitations on the Attainable Intensity of High Power Lasers" // Phys. Rev. Lett., 2010. Vol. 105, no. 8, 080402. 4 pp., arXiv: 1004.5398 [hep-ph].

21. S. A. Smolyansky, A. D. Panferov, A. V. Prozorkevich, M. Bonitz, "Vacuum particle creation under action of a strong external field: an example of irreversible behavior of a system with time reversal symmetry" // p-Adic Numbers Ultrametric Anal. Appl., 2012. Vol. 4, no. 4. Pp. 319-325. 
22. G. V. Dunne, "New strong-field QED effects at extreme light infrastructure" // Eur. Phys. J. D, 2009. Vol. 55, no. 2. Pp. 327-340, arXiv: 0812.3163 [hep-th].

23. A. Casher, H. Neuberger, S. Nussinov, "Chromoelectric Flux Tube Model Of Particle Production" // Phys. Rev. D, 1979. Vol. 20, no.1. Pp. 179-188.

24. J. Schwinger, "Casimir light: a glimpse" // Proc. Nat. Acad. Sci. U.S.A., 1993. Vol. 90, no. 3. Pp. 958-959.

25. А. А. Гриб, С. Г. Мамаев, В. М. Мостепаненко, Вакуумные квантовые эффекты в сильных полях. М.: Энергоатомиздат, 1988. 296 с.; англ. пер.: A. A. Grib, S. G. Mamayev, V. M. Mostepanenko, Vacuum Quantum Effects in Strong Fields. St. Petersburg: Friedmann Lab. Publ., 1994. 361 pp.

26. В. С. Попов, "Рождение пар в переменном внешнем поле (квазиклассическое приближение)" // ЖЭТФ, 1971. Т. 61, № 4. С. 1334-1351; англ. пер.: "Pair production in a variable external field (Quasiclassical approximation)" // Sov. J. Exp. Theor. Phys., 1972. Vol. 34, no. 4. Pp. 709-718.

27. G. J. Troup, H. S. Perlman, "Pair Production in a Vacuum by an Alternating Field" // Phys. Rev. D, 1972. Vol. 6, no. 8. Pp. 2299-2299.

28. В. Б. Берестецкий, Е. М. Лифиии, Л. П. Питаевский, Теоретическая физика. T. IV: Квантовая электродинамика. М.: Наука, 1980. 704 с. [V. B. Beresteckiy, E. M. Lifshits, L. P. Pitaevskiy, Theoretical physics. Vol. IV: Quantum electrodynamics. Moscow: Nauka, 1980. 704 pp.]

29. A. Ringwald, "Pair production from vacuum at the focus of an X-ray free electron laser" // Phys. Lett. B, 2001. Vol.510, no. 1-4. Pp. 107-116, arXiv: hep-ph/0103185.

30. С. Г. Мамаев, Н. Н. Трунов, "Поляризация вакуума и рождение частиц в нестационарном однородном электромагнитном поле" // Яд. физ., 1979. Т. 30, № 5. С. 1301-1311; англ. пер.: S. G. Mamaev, N. N. Trunov, "Vacuum Polarization And Particle Creation In Nonstationary Homogeneous Electromagnetic Field"// Sov. J. Nucl. Phys., 1979. Vol.30, no. 5. Pp. 677.

31. S. Schmidt, D. Blaschke, G. Röpke, S. A. Smolyansky, A. V. Prozorkevich, V. D. Toneev, "A Quantum Kinetic Equation for Particle Production in the Schwinger Mechanism" // Int. J. Mod. Phys. E, 1998. Vol. 07, no. 06. Pp. 709-722, arXiv: hep-ph/9809227.

32. D. B. Blaschke, A. V. Prozorkevich, C. D. Roberts, S. M. Schmidt, S. A. Smolyansky, "Pair Production and Optical Lasers"// Phys. Rev. Lett., 2006. Vol.96, no. 14, 140402. 4 pp., arXiv: nucl-th/0511085.

33. A. V. Filatov, A. V. Prozorkevich, S. A. Smolyansky, "Pair creation by electromagnetic wave in a collisionless plasma" // Proc. SPIE, 2006. Vol. 6165, 616509.

34. F. Hebenstreit, R. Alkofer, G. V. Dunne, H. Gies, "Momentum Signatures for Schwinger Pair Production in Short Laser Pulses with a Subcycle Structure" // Phys. Rev. Lett., 2009. Vol. 102, no. 15, 150404. 4 pp., arXiv: 0901.2631 [hep-ph].

35. C. K. Dumlu, G. V. Dunne, "Stokes Phenomenon and Schwinger Vacuum Pair Production in Time-Dependent Laser Pulses" // Phys. Rev. Lett., 2010. Vol. 104, no. 4, 250402, arXiv: 1004.2509 [hep-th].

36. F. Hebenstreit, R. Alkofer, H. Gies, "Particle Self-Bunching in the Schwinger Effect in Spacetime-Dependent Electric Fields" // Phys. Rev. Lett., 2011. Vol.107, no. 18, 180403. 5 pp., arXiv: 1106.6175 [hep-ph].

37. F. Hebenstreit, Schwinger effect in inhomogeneous electric fields: PhD thesis, 2011. 121 pp., arXiv: 1106.5965 [hep-ph]

38. D. B. Blaschke, V. V. Dmitriev, G. Röpke, S. A. Smolyansky, "BBGKY kinetic approach for an $e^{-} e^{+} \gamma$ plasma created from the vacuum in a strong laser-generated electric field: The one-photon annihilation channel" // Phys. Rev. D, 2011. Vol. 84, no. 8, 085028. 13 pp., arXiv: 1105.5397 [hep-ph]. 
MSC: $81 \mathrm{V10}$

SOME PROBLEMS OF THE KINETIC DESCRIPTION OF VACUUM PARTICLE CREATION IN STRONG FIELDS

\section{S. A. Smolyansky ${ }^{1}$, A. V. Prozorkevich ${ }^{1}$, M. Bonit $z^{2}$}

1 N. G. Chernyshevsky Saratov State University (National Research University),

Faculty of Mathematics and Mechanics,

83, Astrakhanskaya st., Saratov, 410012, Russia.

2 Institut für Theoretische Physik und Astrophysik der Christian-Albrechts-Universität zu Kiel, Germany, 24098 Kiel, Leibnizstrasse, 15.

E-mails: smol@sgu.ru, avproz@yandex.ru, bonitz@theo-physik.uni-kiel.de

Some of the actual problems in the theory of vacuum particle creation in strong field are discussed.

Key words: vacuum pair creation, kinetic equation, perturbation theory.

Original article submitted 16/XI/2012; revision submitted $27 / \mathrm{III} / 2013$.

Stanislav A. Smolyansky (Dr. Sci (Phys. \& Math.)), Professor, Dept. of Theoretical Physics. Alexander V. Prozorkevich (Dr. Sci (Phys. \& Math.)), Professor, Dept. of General Physics. Michael Bonitz, Professor, Dept. of Statistical Physics. 